\title{
UV star-formation rates of GRB host galaxies
}

\author{
L. Christensen ${ }^{1}$, J. Hjorth ${ }^{2}$, and J. Gorosabel $\mathrm{l}^{3,4,5}$ \\ 1 Astrophysikalisches Institut Potsdam, An der Sternwarte 16, 14482 Potsdam, Germany \\ e-mail: 1christensen@aip.de \\ ${ }^{2}$ Niels Bohr Institute, Astronomical Observatory, Juliane Maries Vej 30, 2100 Copenhagen, Denmark \\ 3 Danish Space Space Research Institute, Juliane Maries Vej 30, 2100 Copenhagen Ø, Denmark \\ 4 Space Telescope Science Institute, 3700, San Martin Drive, Baltimore, MD 21218, USA \\ 5 Instituto de Astrofísica de Andalucía, IAA-CSIC, Granada, Spain
}

Received 1 March 2004 / Accepted 25 June 2004

\begin{abstract}
We study a magnitude-limited sample of 10 gamma-ray burst (GRB) host galaxies with known spectroscopic redshifts $(0.43<z<2.04)$. From an analysis of the spectral energy distributions (SEDs), based on published broad-band optical and near-infrared photometry, we derive photometric redshifts, galaxy types, ages of the dominant stellar populations, internal extinctions, and ultraviolet (UV) star-formation rates (SFRs) of the host galaxies. The photometric redshifts are quite accurate despite the heterogeneous nature of the sample: the rms errors are $\sigma(z)=0.21$ and $\sigma\left(\Delta z /\left(1+z_{\text {spec }}\right)\right)=0.16$ with no significant systematic offsets. All the host galaxies have SEDs similar to young starburst galaxies with moderate to low extinction. A comparison of specific SFRs with those of high-redshift galaxies in the Hubble Deep Fields shows that GRB hosts are most likely similar to the field galaxies with the largest specific SFRs. On the other hand, GRB hosts are not significantly younger than starburst field galaxies at similar redshifts, but are found to be younger than a sample of all types of field galaxies.
\end{abstract}

Key words. galaxies: high-redshift - galaxies: starburst - gamma rays: bursts

\section{Introduction}

The association of Gamma-Ray Bursts (GRBs) with collapsing massive stars has been inferred through many observations during the past 7 years, notably through supernova bumps in the afterglow light curves or localisations of the afterglow close to star forming regions (Castro-Tirado \& Gorosabel 1999; Bloom et al. 1999; Fruchter et al. 1999a; Galama et al. 1998; Kulkarni et al. 1998; Holland \& Hjorth 1999; Fynbo et al. 2000). Additionally, Bloom et al. (2002) argued that GRB progenitors, given their small positional offsets relative to their hosts, are likely massive stars. Evidence that at least some longduration GRBs are associated with supernovae came with the observations of the afterglow of GRB 030329, which showed distinct spectral supernova features (Stanek et al. 2003; Hjorth et al. 2003b).

Underlying host galaxies have been found in all cases where GRBs were localised with sub-arcsecond precision. Currently, the sample consists of $\sim 35$ such hosts with known redshifts in the range $0.1055<z<4.5$ (Prochaska et al. 2004; Andersen et al. 2000). The reported magnitudes of the hosts are $20.4<R<30$ (Malesani et al. 2004; Jaunsen et al. 2003).

The life time of the massive stars believed to produce longduration GRBs is of the order of a few Myr. If the host galaxies are indeed forming such massive stars this should be reflected in their spectral energy distributions (SEDs) which, in the absence of reddening, should reveal a blue continuum. Moreover, their star-formation rates (SFRs) should be large. The integrated SFR of a galaxy can be found from measurements of $\mathrm{H} \alpha$ or [O II] line fluxes, or from measuring the flux in the UV continuum at $1500-2800 \AA$ in the rest frame of the galaxy (Kennicutt 1998). The faintness of GRB hosts presents a problem for spectroscopy as they require long integration times on the largest telescopes. Ground-based photometry in several filters presents an alternative possibility for studying the SEDs of faint hosts. From such SEDs the UV continuum flux can be determined and the galaxy type can be inferred. Previous investigations have shown that GRB hosts have SEDs similar to starburst galaxies (Sokolov et al. 2001; Gorosabel et al. 2003a,b; Christensen et al. 2004), and their SFRs inferred from optical methods are moderate $<10 M_{\odot} \mathrm{yr}^{-1}$ (Fruchter et al. 1999a; Bloom et al. 1998; Djorgovski et al. 1998, 2001a). Larger SFRs have been reported based on spectroscopic measurements. The GRB 000418 host has an un-obscured SFR of $55 M_{\odot} \mathrm{yr}^{-1}$ derived from the [O II] line flux (Bloom et al. 2003). All the optical methods for determining the SFRs are affected by dust extinction in the hosts. Therefore, the optically inferred SFRs represent lower limits to the true SFRs. Radio and sub-mm data are much less affected by dust extinction, and observations of GRB hosts indicate that the unextincted SFRs can be as much as two orders of magnitude larger than those derived from optical estimators (Berger et al. 2001; Berger et al. 2003; 
Tanvir et al. 2004). However, not all hosts have very large SFRs; some have SFRs $<200 M_{\odot} \mathrm{yr}^{-1}$ suggested by radio observations (Vreeswijk et al. 2001).

The SFRs of individual GRB hosts published in the literature have been argued to be comparable to those of other high redshift galaxies selected by optical methods. Djorgovski et al. (2001b) found that the [O II] equivalent widths of GRB hosts are somewhat larger than that of field galaxies at similar redshifts as GRBs. Likewise, Fruchter et al. (1999b) found that a sample of three GRB hosts has bluer colours on average than field galaxies in the Hubble Deep Field.

In this paper we present a statistical analysis of the properties of GRB hosts as compared with other high redshift galaxies. We present the $10 \mathrm{GRB}$ hosts selected for the analysis in Sect. 2, the derived photometric redshifts in Sect. 3, and the SED investigations in Sect. 4. We estimate the SFRs of the 10 GRB hosts by computing the rest frame UV flux in Sect. 5. Since the absolute luminosity of the hosts vary by a large factor we also analyse the specific SFRs normalised by the host luminosities. Comparisons with properties of field galaxies selected from the Hubble Deep Field are presented in Sect. 6. Our results are discussed and summarised in Sect. 7.

We assume a flat cosmological model with $\Omega_{\mathrm{m}}=0.3$, $\Omega_{\Lambda}=0.7$ and $H_{0}=65 \mathrm{~km} \mathrm{~s}^{-1} \mathrm{Mpc}^{-1}$. The choice of parameters affects the luminosity distance of the galaxies, and thereby the derived SFRs.

\section{GRB host galaxy sample}

Our own multi-colour imaging studies of GRB host galaxies have been presented elsewhere (Gorosabel et al. 2003a,b; Christensen et al. 2004). The present work is based on a compilation of photometry already available in the literature (including our own observational work).

We imposed a magnitude limit to make sure that the hosts entering the sample were bright enough to have fairly accurate multi-colour photometry in at least 5 optical and near-IR bands. This implies that the sample is limited by the available multi-colour photometry from the literature. However, with a maximum magnitude of $R=25.3$ the sample is magnitudelimited. These criteria also implied that no host of a GRB occurring after 2002 is included due to poor multi-colour sampling. We also required that a redshift for the host galaxy or the afterglow be known. Finally, we excluded a few GRB hosts which had such complex morphologies that the resulting SEDs might be dominated by different sub-components at different wavelengths (such as the GRB 980613, GRB 011121, and GRB 011211 hosts). Having a complex morphology, the GRB 980613 host shows colour variations in HST/STIS images of $m_{\mathrm{CL}}-m_{\mathrm{LP}}>0.7$ between individual components (Hjorth et al. 2002). Similar colour variations was found in ground based observations by Djorgovski et al. (2003). Such variations in colours make any detailed analysis of the overall SED subject to great uncertainty in terms of the derived extinction and age. Also the GRB 011121 and GRB 011211 hosts have complex surroundings, but not much is presently known about the colours of the hosts themselves (Garnavich et al. 2003; Greiner et al. 2003; Jakobsson et al. 2003). We therefore chose to exclude these hosts, but note that these systems represent likely mergers which may show significant star formation. Images of the various hosts are presented elsewhere (Bloom et al. 2002; Djorgovski et al. 2003; Castro et al. 2003).

To summarize, our selection criteria are:

- Detection in 5 or more filters;

$-R<25.3$

- Known redshift;

- Not very complex morphology.

These criteria limited our investigations to $10 \mathrm{GRB}$ hosts in the redshift range $0.433<z<2.037$ with a mean and median redshifts of $z=0.97$ and $z=0.85$, somewhat smaller than those derived from the $35 \mathrm{GRB}$ redshifts measured to date $(z=1.43$ and $z=1.10)^{1}$. The sample is presented in Table 1 .

Some of the host magnitudes were obtained a few weeks after the burst when the optical afterglow could still contaminate the observed flux. In these cases the host magnitudes are derived from fits to the light curves of the afterglows. Since the light curve of the afterglow can be described by a power law, the total flux is given by $f(t)=f_{0} \times t^{-\alpha}+f_{\text {host }}$, where the first term characterizes the fading afterglow. If the light curve is well sampled, the flux of the host $f_{\text {host }}$ can be estimated. For example, the GRB 980703 host magnitudes were derived this way in Vreeswijk et al. (1999). Data obtained more than one year after this particular burst gave magnitudes which are consistent with those reported in Table 1 (Holland et al. 2001). As another example, the expected $B$ band magnitude of the GRB 010921 afterglow would be 3 mag fainter than the host magnitude reported in Price et al. (2002) at the time of the observations 21 days after the burst.

Ideally, the magnitudes of a host should be derived using one consistent photometric technique for all filters. For example, in the case of aperture photometry the magnitudes should be derived using the same aperture. We can not be sure that this is the case for the magnitudes given in Table 1. In the cases where the hosts are more extended than point sources the effect should be negligible as long as the authors have applied a large enough aperture for deriving the host magnitudes.

Furthermore, one should note that we have restricted ourselves to analysing only the bright end of the luminosity function since it is easier to perform multiband observations of the brightest hosts. Only little is known about the nature of the fainter host galaxies (Berger et al. 2002; Jaunsen et al. 2003; Hjorth et al. 2003a).

\section{Photometric redshifts}

The magnitudes in Table 1 were used for comparison with theoretical galaxy template spectra from Bruzual \& Charlot (1993). This was done using the program Hyper $Z^{2}$ developed by Bolzonella et al. (2000).

The templates which are used to fit the GRB host magnitudes consist of elliptical, different types of spiral galaxies, irregular, and starburst spectra at various ages having different

1 GRB redshifts can be found at Jochen Greiner's web-pages: http: //www .mpe.mpg.de/ jcg/grbgen.html

2 http://webast.ast.obs-mip.fr/hyperz/ 
Table 1. Magnitudes in the Vega system in various filters for the selected sample of 10 GRB hosts taken from the literature. The magnitudes are not corrected for Galactic extinction. ${ }^{\dagger}$ indicates that the host magnitudes were derived from power law fits to the afterglow light curves.

\begin{tabular}{|c|c|c|c|}
\hline Host & Filter (Mag) & Telescope & Reference \\
\hline GRB 970228 & $\begin{array}{l}B>26.08 \\
V=25.77 \pm 0.2 \\
R_{\mathrm{c}}=25.22 \pm 0.2 \\
I=24.4 \pm 0.2 \\
H=23.2 \pm 0.3 \\
K=22.6 \pm 0.2\end{array}$ & $\begin{array}{l}\text { VLT } \\
\text { HST/STIS } \\
\text { HST/STIS } \\
\text { HST/WFPC2 } \\
\text { HST/NICMOS2 } \\
\text { NIRC/Keck I }\end{array}$ & $\begin{array}{l}\text { Sokolov et al. (2001) } \\
\text { Galama et al. (2000) } \\
\text { Fruchter et al. (1999a) } \\
\text { Chary et al. (2002) }\end{array}$ \\
\hline GRB 970508 & $\begin{array}{l}B=25.89 \pm 0.19 \\
V=25.34 \pm 0.22 \\
R_{\mathrm{c}}=25.06 \pm 0.17 \\
I_{\mathrm{c}}=24.11 \pm 0.25 \\
K=22.7 \pm 0.2\end{array}$ & $\begin{array}{l}\text { BTA } \\
\text { BTA } \\
\text { BTA } \\
\text { BTA } \\
\text { Keck I/NIRC }\end{array}$ & Chary et al. (2002) \\
\hline GRB 980703 & $\begin{array}{l}B=23.40 \pm 0.12 \\
V=23.04 \pm 0.08^{\dagger} \\
R=22.58 \pm 0.06 \\
I=21.95 \pm 0.25 \\
J=20.87 \pm 0.11 \\
H=20.27 \pm 0.19 \\
K=19.62 \pm 0.12\end{array}$ & BTA & $\begin{array}{l}\text { Sokolov et al. (2001) } \\
\text { Vreeswijk et al. (1999) }\end{array}$ \\
\hline GRB 990123 & $\begin{array}{l}U=23.6 \pm 0.15 \\
B=24.23 \pm 0.1^{\dagger} \\
V=24.20 \pm 0.15 \\
R=23.77 \pm 0.1 \\
I=23.65 \pm 0.15 \\
K=21.7 \pm 0.3\end{array}$ & Keck I/NIRC & Castro-Tirado et al. (1999) \\
\hline GRB 990712 & $\begin{array}{l}U=23.12 \pm 0.05 \\
B=23.36 \pm 0.09 \\
V=22.39 \pm 0.03 \\
R=21.84 \pm 0.02 \\
I=21.41 \pm 0.03 \\
J=20.81 \pm 0.17 \\
H=20.25 \pm 0.19 \\
K S=20.05 \pm 0.1\end{array}$ & $\begin{array}{l}\text { ESO-3.6 m } \\
\text { DK-1.5 m/DFOSC } \\
\text { DK-1.5 m/DFOSC } \\
\text { DK-1.5 m/DFOSC } \\
\text { DK-1.5 m/DFOSC } \\
\text { NTT/SOFI } \\
\text { NTT/SOFI }\end{array}$ & Le Floc'h et al. (2003) \\
\hline GRB 991208 & $\begin{array}{l}B=25.19 \pm 0.17^{\dagger} \\
V=24.55 \pm 0.16 \\
R=24.26 \pm 0.15 \\
I=23.3 \pm 0.2 \\
K=21.7 \pm 0.2\end{array}$ & NIRC/Keck I & $\begin{array}{l}\text { Castro-Tirado et al. (2001) } \\
\text { Chary et al. (2002) }\end{array}$ \\
\hline
\end{tabular}

star-formation histories. The SFRs decrease with time, such that $\operatorname{SFR} \propto \exp (-t / \tau)$, where the characteristic timescale, $\tau$ increases along the Hubble sequence. The starburst template is created as an instantaneous burst of star formation $(\tau \rightarrow 0)$, which instantly uses up all available gas, while an irregular Im template has a constant SFR $(\tau \rightarrow \infty)$. We used the Miller $\&$ Scalo (1979) initial mass function (IMF) for calculating the templates, as well as a Salpeter (1955) IMF with stellar masses between 0.1 and $125 M_{\odot}$. The Miller \& Scalo IMF produces fewer massive stars compared to a Salpeter IMF and is flatter below $1 M_{\odot}$. The metallicities of the templates were solar, $Z=0.02$.

Applying an additional extinction term to the templates, the fitting allows an estimate of the type of galaxy, age, and the intrinsic extinction $\left(A_{\mathrm{V}}\right)$ for the galaxies. In the fits we used the extinction curve estimated for starburst galaxies
(Calzetti et al. 2000). We also analysed the SEDs using other extinction curves, e.g. from the Milky Way (Seaton 1979), the Large Magellanic Cloud (Fitzpatrick 1986), and the Small Magellanic Cloud (Prevot et al. 1984). We found that the derived ages for the dominant population of stars and extinctions did not depend on the chosen extinction curve. The reason is that actual values of the extinctions are quite small $\left(A_{\mathrm{V}}<1\right)$, thus the various extinction curves produce minor differences, as quantified in Sect. 4.1.

A direct application of HyperZ results in the photometric redshifts listed in Table 2 . In most cases these are consistent with the spectroscopic ones. The mean value and standard deviation of $\left(z_{\text {phot }}-z_{\text {spec }}\right)$ is -0.04 and 0.21 , respectively. Only for the GRB 990123 host the redshift estimate is inaccurate $(\Delta z>0.3)$, but taking into account the uncertainty of the estimate the difference is only $1.6 \sigma$. A reason for the 
Table 1. continued.

\begin{tabular}{|c|c|c|c|}
\hline Host & Filter (Mag) & Telescope & Reference \\
\hline GRB 000210 & $\begin{array}{l}U=23.54 \pm 0.13 \\
B=24.40 \pm 0.13 \\
V=24.22 \pm 0.08 \\
R=23.46 \pm 0.10 \\
I=22.49 \pm 0.12 \\
Z=22.83 \pm 0.28 \\
J \mathrm{~S}=21.98 \pm 0.10 \\
H=21.51 \pm 0.23 \\
K \mathrm{~S}=20.94 \pm 0.14\end{array}$ & $\begin{array}{l}\text { ESO-3.6 m/EFOSC2 } \\
\text { ESO-3.6 m/EFOSC2 } \\
\text { ESO-3.6 m/EFOSC2 } \\
\text { VLT/FORS1 } \\
\text { ESO-3.6 m/EFOSC2 } \\
\text { DK-1.5 m/DFOSC } \\
\text { VLT/ISAAC } \\
\text { NTT/SOFI } \\
\text { VLT/ISAAC }\end{array}$ & $\begin{array}{l}\text { Gorosabel et al. (2003a) } \\
\text { Piro et al. (2002) } \\
\text { Gorosabel et al. (2003a) }\end{array}$ \\
\hline GRB 000418 & $\begin{array}{l}U=23.54 \pm 0.3 \\
B=24.07 \pm 0.05 \\
V=23.80 \pm 0.06 \\
R=23.36 \pm 0.05 \\
I=22.79 \pm 0.05 \\
Z=22.46 \pm 0.1 \\
J \mathrm{~s}=22.27 \pm 0.1 \\
K \mathrm{~s}=21.19 \pm 0.3\end{array}$ & $\begin{array}{l}\text { ESO-3.6 m/EFOSC2 } \\
\text { NOT/ALFOSC } \\
\text { NOT/ALFOSC } \\
\text { NOT/ALFOSC } \\
\text { NOT/ALFOSC } \\
\text { NOT/ALFOSC } \\
\text { VLT/ISAAC } \\
\text { VLT/ISAAC }\end{array}$ & Gorosabel et al. (2003b) \\
\hline GRB 000926 & $\begin{array}{l}B=25.49 \pm 0.33^{\dagger} \\
V=25.08 \pm 0.06 \\
R \mathrm{c}=24.83 \pm 0.07 \\
I \mathrm{c}=24.59 \pm 0.01 \\
J_{\mathrm{AB}}=24.1_{-0.4}^{+0.7}\end{array}$ & & $\begin{array}{l}\text { Castro et al. (2003) } \\
\text { J. Fynbo (priv. comm.) }\end{array}$ \\
\hline GRB 010921 & $\begin{array}{l}B=23.42 \pm 0.08 \\
V=22.32 \pm 0.06 \\
R \mathrm{c}=21.93 \pm 0.09 \\
I \mathrm{c}=21.05 \pm 0.08\end{array}$ & & $\begin{array}{l}\text { Price et al. (2002) } \\
\text { Park et al. (2002) }\end{array}$ \\
\hline & $\begin{array}{l}J=20.34 \pm 0.02 \\
H=19.75 \pm 0.04 \\
K S=19.07 \pm 0.04\end{array}$ & $\begin{array}{l}\text { Keck I/NIRC } \\
\text { Keck I/NIRC } \\
\text { Keck I/NIRC }\end{array}$ & Price et al. (2002) \\
\hline
\end{tabular}

Table 2. Results from the best fits from HyperZ. Column 2 gives the spectroscopic redshift of the hosts, which was held fixed while running HyperZ. Column 3 lists the unconstrained photometric redshifts and the associated 68\% confidence levels. The best fit template is given in Col. 4, and the corresponding ages and internal extinctions are given in Cols. 5 and 6, respectively, using a Miller \& Scalo IMF. Columns 8 and 9 list the results of the extinction and $\chi^{2} /$ d.o.f. for a Salpeter IMF.

\begin{tabular}{ccccccc|cc}
\hline \hline \multicolumn{7}{c|}{ Miller \& Scalo IMF } & \multicolumn{2}{c}{ Salpeter IMF } \\
\hline Host & $z_{\text {spec }}$ & $z_{\text {phot }}$ & Spectrum & Age $(\mathrm{Gyr})$ & $A_{\mathrm{V}}$ & $\chi^{2} /$ d.o.f. & $A_{\mathrm{V}}$ & $\chi^{2} /$ d.o.f. \\
\hline GRB 970228 & 0.695 & $0.78_{-0.12}^{+0.24}$ & burst & $0.064 \pm 0.012$ & $0.12_{-0.12}^{+0.18}$ & 0.228 & $0.07_{-0.07}^{+0.16}$ & 0.239 \\
GRB 970508 & 0.835 & $0.87_{-0.12}^{+0.07}$ & burst & $0.091 \pm 0.090$ & $0.17_{-0.17}^{+0.11}$ & 0.146 & $0.17_{-0.17}^{+0.11}$ & 0.082 \\
GRB 980703 & 0.966 & $0.82_{-0.18}^{+0.16}$ & burst & $0.006 \pm 0.122$ & $1.60_{-1.27}^{+0.05}$ & 0.683 & $0.38_{-0.24}^{+0.25}$ & 0.831 \\
GRB 990123 & 1.600 & $2.18_{-0.40}^{+0.31}$ & Sa & $0.012 \pm 0.006$ & $0.90_{-0.20}^{+0.17}$ & 0.823 & $0.96_{-0.23}^{+0.05}$ & 0.773 \\
GRB 990712 & 0.433 & $0.42_{-0.01}^{+0.003}$ & burst & 0.255 & $0.15_{-0.05}^{+0.04}$ & 2.815 & $0.15_{-0.05}^{+0.06}$ & 0.959 \\
GRB 991208 & 0.706 & $0.78_{-0.08}^{+0.02}$ & burst & $0.091 \pm 0.090$ & $0.35_{-0.35}^{+0.18}$ & 0.621 & $0.37_{-0.30}^{+0.16}$ & 0.613 \\
GRB 000210 & 0.846 & $0.84_{-0.04}^{+0.05}$ & burst & 0.181 & $0.02_{-0.02}^{+0.06}$ & 1.225 & $0.06_{-0.06}^{+0.08}$ & 1.105 \\
GRB 000418 & 1.118 & $1.00_{-0.003}^{+0.02}$ & burst & $0.064 \pm 0.027$ & $0.12_{-0.12}^{+1.50}$ & 2.448 & $0.14_{-0.14}^{+1.40}$ & 2.266 \\
GRB 000926 & 2.037 & $2.09_{-0.59}^{+0.54}$ & burst & $0.091 \pm 0.060$ & $0.00^{+0.44}$ & 0.020 & $0.02_{-0.02}^{+0.43}$ & 0.025 \\
GRB 010921 & 0.451 & $0.37_{-0.02}^{+0.02}$ & burst & $0.091 \pm 0.090$ & $0.68_{-0.02}^{+0.05}$ & 2.361 & $0.69_{-0.27}^{+0.05}$ & 2.353 \\
\hline
\end{tabular}

relatively large discrepancy could be that the Balmer jump is not well sampled. Indeed, very accurate photometric redshifts can be determined if the photometric uncertainties are small and the Balmer jump is well sampled as is the case for the GRB 000418, GRB 000210, and GRB 990712 hosts.
At higher redshifts the broad band filters cover a narrower wavelength range of the rest frame spectrum due to the factor $(1+z)$ accounting for the cosmological expansion. Therefore, the accuracy of photometric redshift estimates is expected to decrease with increasing redshift. A different 
measure of the accuracy can be obtained by calculating the expression $\Delta z=\left(z_{\text {phot }}-z_{\text {spec }}\right) /\left(1+z_{\text {spec }}\right)$ for the sample of GRB hosts. We find a mean value of 0.015 and a standard deviation of 0.16 using this expression, showing that it is possible to determine photometric redshifts accurately for GRB hosts. For comparison, Fernández-Soto et al. (2001) derived more accurate photometric redshifts for galaxies observed through 7 bands in the Hubble Deep Field, having a standard deviation of 0.065 , which is likely due to smaller photometric uncertainties for their galaxies. While the average number of bands of observations of the GRB host sample is 6.6, 3 of the hosts have observations in 5 filters only. Additionally, the standard broad band filters used for ground based photometry are not optimally tuned to find photometric redshifts. We find no outliers for the estimation of photometric redshifts, indicating that this technique is robust for estimating redshifts of GRB hosts.

\section{Spectral energy distributions}

While HyperZ was written for obtaining photometric redshifts of galaxies in large surveys, it also serves the purpose of finding the best matching theoretical galaxy template for a given set of broad-band observations. In the remainder of this work we shall fix the redshifts of the GRB hosts to the values given by the spectroscopic measurements. This was done in order to optimise the estimates of other output parameters, as explained below.

The best fit is obtained by minimizing the expression

$\chi^{2}=\sum_{i}\left(\frac{F_{\mathrm{host}, i}-k \times F_{\mathrm{temp}, i}}{\sigma\left(F_{\mathrm{host}, i}\right)}\right)^{2}$

where the sum is to be taken over all filters, $i . F_{\text {host }}$ is the flux density of the host in the filter $i, \sigma\left(F_{\text {host }, i}\right)$ is the associated error, and $k$ is a normalization constant. $F_{\text {temp, } i}$ is the flux of the template in the filter $i$, which is calculated using the throughput for the given filter and instrument. HyperZ provides a data set of throughputs for many instruments. For the instruments where we had no knowledge of the throughput (as for example for the Russian 6 m-BTA telescope), we simply used the throughput of the given filter used for the observation, i.e. without convolving with the quantum efficiency of the CCD. We tested to see if it had any significance using e.g. the VLT throughput curve for the given filter and found the effect to be negligible. Since the photometric errors are large in these cases, the results for the best fit template did not change. In all cases, only templates from one single burst of star formation were used. This is a simplification since more than one population of stars may be present in the hosts (Sokolov et al. 2001; Christensen et al. 2004). Hereafter the derived stellar properties will refer exclusively to the dominant population.

Detailed analyses of the SEDs of the host galaxies of GRB 000210, GRB 000418, and GRB 990712 are presented by Gorosabel et al. (2003a), Gorosabel et al. (2003b), and Christensen et al. (2004), respectively. Similar thorough individual SED analyses are beyond the scope of this paper.

Results from the SED fittings are given in Table 2. Column 2 gives the measured spectroscopic redshift, which was held fixed while running HyperZ. For comparison the unconstrained photometric redshifts are listed in Col. 3. In Col. 4 the best fit templates are given, in Col. 5 the age of the template, Col. 6 gives the extinction, and Col. 7 lists the reduced $\chi^{2}$ per degree of freedom (d.o.f.) for the best fits. All these values are derived using templates constructed using a Miller \& Scalo IMF. Columns 8 and 9 list the extinction and $\chi^{2} /$ d.o.f. for fits using a Salpeter IMF. The age and galaxy type do not change choosing a different IMF, and all the observed host SEDs are well fit by starburst templates or young star-forming galaxy types.

Generally, the ages of the dominant population of stars are smaller than 0.2 Gyr for all GRB hosts, and the extinctions found for the systems are relatively small: $0<A_{\mathrm{V}}<2$. Plots of the best fit templates for each host are shown in Fig. 1.

\subsection{Ages and extinctions}

To estimate errors on ages and extinctions we analysed the values of $\chi^{2}$ for SED fits of various templates while varying the extinction. We derived errors of the extinction and age on the basis on which fits gave an increase in the $\chi^{2}$ by $\Delta\left(\chi^{2}\right)=1$. The host of GRB 000418 and GRB 980703 are treated separately below.

Using different extinction laws resulted in differences in $A_{\mathrm{V}}$ smaller than 0.1 , and furthermore, the estimated ages remained constant for any applied extinction law.

Since GRBs are thought to be produced by the most massive stars, ages much larger than $10 \mathrm{Myr}$ of the burst population are inconsistent with this hypothesis. However, we can not exclude the possibility that two separate bursts of star formation would be able to reproduce the SEDs of the hosts. The burst template model uses up all gas in the first burst, while a more reliable model would likely have $\tau>0$. We have fit all host magnitudes to Im models, which generally produced acceptable fits $\left(\chi^{2} /\right.$ d.o.f. $\left.<2\right)$. However, this was not the case for the GRB 990712, GRB 000210, and GRB 000418 hosts, which gave $\chi^{2} /$ d.o.f. $=14,24$, and 6 , respectively. These hosts are the only ones which have extensive multiband photometric measurements, and their SEDs are well constrained. This implies that a continuous SFR is not in agreement with the observed SED. The large errors for the photometry of the other hosts make their SEDs less well constrained.

A special comment is needed for the host of GRB 000418. In Gorosabel et al. (2003b), the final reported results for the SED fits are consistent with those reported here in Table 2. However, the actual best fit having the minimum $\chi^{2} /$ d.o.f. for a Salpeter IMF and a Calzetti extinction law, is obtained with a $0.004 \mathrm{Gyr}$ old starburst template with an extinction of $A_{\mathrm{V}}=1.38$ (see Table 3 in Gorosabel et al. 2003b). We will return to the implication of this uncertainty in Sect. 6.4.

As seen in Table 2 the estimated extinction for the GRB 980703 host changes by a large factor for the two applied IMFs. However, the lower limit on the extinction derived for the Miller \& Scalo IMF fit is consistent with that derived for the Salpeter IMF fit. 

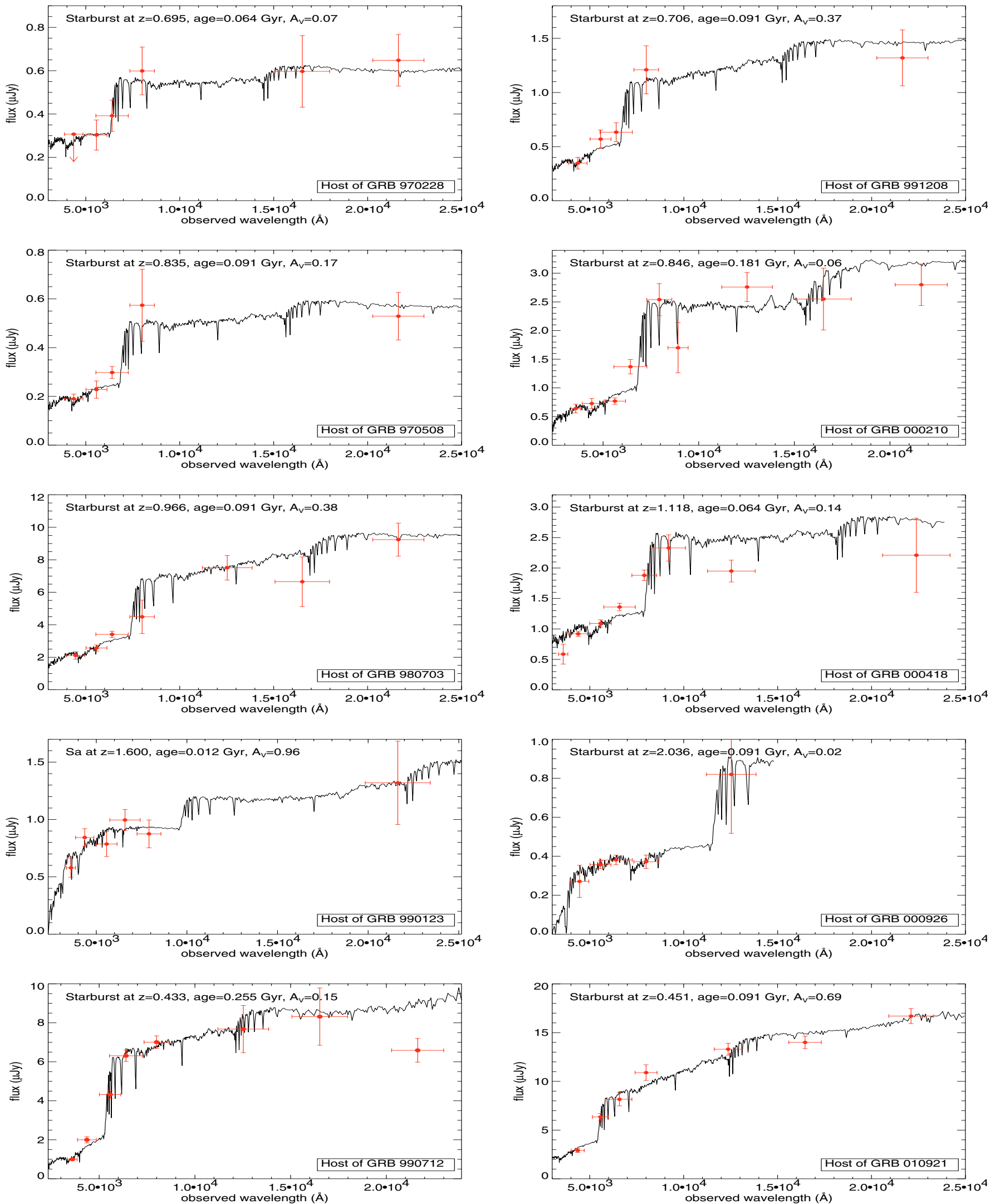

Fig. 1. Best fits of the 10 GRB host SEDs fitted to synthetic spectra based on a Salpeter IMF as calculated using HyperZ. Redshifts, type of the template, and the extinction are given as inserts in the plots. The solid circles show the available photometry for each host (see Table 1) after correcting for Galactic extinction. The associated horizontal error bars denote the FWHM of the filters. 
Table 3. Ages in Myr derived for fits to various metallicities. In addition to the redshifts, also extinction values are held fixed.

\begin{tabular}{lllll}
\hline \hline Host & $A_{\mathrm{V}}$ (fixed) & $Z=0.02$ & $Z=0.008$ & $Z=0.004$ \\
\hline GRB 970228 & 0.07 & 100 & 50 & 50 \\
GRB 970508 & 0.17 & 100 & 100 & 100 \\
GRB 980703 & 0.38 & 100 & 100 & 100 \\
GRB 990123 & 0.96 & 5 & 5 & 5 \\
GRB 990712 & 0.15 & 200 & 200 & 200 \\
GRB 991208 & 0.37 & 100 & 100 & 100 \\
GRB 000210 & 0.06 & 200 & 200 & 200 \\
GRB 000418 & 0.14 & 70 & 100 & 100 \\
GRB 000926 & 0.02 & 100 & 100 & 100 \\
GRB 010921 & 0.69 & 100 & 100 & 100 \\
\hline
\end{tabular}

\subsection{Ages and metallicities}

There is a well known degeneracy between the age of a stellar population and the metallicity for a given SED. In order to quantify how much this degeneracy affects our results we fitted the SEDs to starburst templates, created from a Salpeter IMF, with metallicities of $1,0.4$, and 0.2 times solar, respectively, using the GALAXEV library of evolutionary stellar population synthesis models (Bruzual \& Charlot 2003). The ages of the templates were between $5 \mathrm{Myr}$ and $200 \mathrm{Myr}$. While keeping the extinction values fixed to those obtained in Table 2 the best fit ages for the different metallicities are listed in Table 3 . Compared to the ages found by HyperZ in Table 2, there are only small differences which are likely due to the differences for the input templates. Moreover, there is a general agreement between the best fit ages for the various metallicities. Because of this result we estimate that for the GRB hosts the age-metallicity degeneracy produces small systematic errors.

Alternatively, as shown from the analysis of the GRB 000418 host in Gorosabel et al. (2003b), the metallicity is not strongly constrained in the case where the SED is analysed through broad band magnitudes.

For the median redshift $z \approx 1$ of the GRB hosts our SED analysis covers the rest-frame far-UV to rest-frame $J$ bands. Using SED analyses of star clusters in the nearby NGC 3310 starburst galaxy de Grijs et al. (2003) find that the ages are well reproduced when UV-near-IR pass bands are included, which supports our finding of a consistent best fit age.

\section{Star-formation rates}

Since mostly young stars contribute to the UV flux in a galaxy there is a relation between the rest frame UV continuum flux of a galaxy and the unobscured SFR. One can estimate the SFR from the rest frame flux at $2800 \AA$ using the relation in Kennicutt (1998)

$\operatorname{SFR}\left(M_{\odot} \mathrm{yr}^{-1}\right)=1.4 \times 10^{-28} L_{\gamma, \mathrm{UV}} \quad\left[\mathrm{erg} \mathrm{s}^{-1} \mathrm{~Hz}^{-1}\right]$.

This method is appropriate for obtaining the SFR as long as there is a continuous formation of stars. It is a good estimator for ages larger than $10^{8}$ years, but shows limitations for ages smaller than $10^{7}$ years. Having found that the ages of some of the GRB hosts could be smaller than $10^{7}$ years, the relation (2) is not always applicable. For younger populations the constant linking the SFR with the luminosity is significantly lower, yielding a smaller SFR for a given flux. On the other hand, dust is expected to be present in star-forming regions in which case the observed flux must be corrected for dust extinction. These two effects have an opposite impact on the derived SFRs.

The method applied to calculate the SFRs from the observed broad band magnitudes was as follows. First the magnitudes were corrected for Galactic extinction using the dust maps of Schlegel et al. (1998). Magnitude offsets calculated by HyperZ from standard filters to the $\mathrm{AB}$ system were added. The $\mathrm{AB}$ system is defined as $m_{\mathrm{AB}}=-2.5 \log f_{v}-48.6$ where $f_{v}$ is the flux density measured in erg $\mathrm{cm}^{-2} \mathrm{~s}^{-1} \mathrm{~Hz}^{-1}$. Broad band magnitudes were converted to flux units (in $\mu \mathrm{Jy}$ ) using the expression $f_{v}=10^{-0.4\left(m_{\mathrm{AB}}-23.9\right)}$. The fluxes at the observed wavelengths $2800(1+z) \AA$ were estimated assuming power law spectra, $f_{v}=f_{0}\left(v / v_{0}\right)^{\beta}$ for the hosts, between the two filters bracketing the observed wavelength at $2800(1+z) \AA$.

The calculated SFRs for the 10 GRB hosts are listed in Table 4. Knowing the extinction of the hosts from the SED analyses, one can correct the SFRs for the effects of extinction. Using the extinction curve of Calzetti et al. (2000) we derive the unextincted SFRs given in Col. 6.

The errors of the SFRs in Table 4 are due to the photometric errors used for interpolation which translates into errors of the $2800 \AA$ A fluxes. The intrinsic scatter in the calibration converting UV flux into SFR is of the order of $30 \%$ (Kennicutt 1998). This uncertainty is not included in the quoted SFRs.

For comparison, SFRs derived from spectroscopic measurements of the [O II] line flux are listed in Col. 7 in Table 4. Apart from the GRB 000418 and GRB 991208 hosts the agreement between the unextincted, UV based and [O II] based SFRs is rather good which suggests that extinction does not play a major role. Values of the SFR derived either from sub-mm, or radio observations are also listed in Table 4 . These measurements generally show larger values, which may indicate very obscured components with no (or faint) optical emission within the galaxies.

One immediately sees from Table 4 that the SFRs of the hosts are moderate, in line with the conclusions of Fruchter et al. (1999a); Djorgovski et al. (2003, 2001a); Castro-Tirado et al. (2001). In Fig. 2 the calculated SFRs are plotted as a function of redshift. Since the faint, high-redshift hosts do not have multiband observations, and therefore are not studied here, the trend for larger SFRs at high redshifts may be caused by the selection of intrinsically bright hosts.

For the GRB 000926 host at $z=2.037$ and the GRB 990123 host at $z=1.600$ we use the observed $B$ and $U$ band magnitudes, respectively, as a rough measure of their continuum flux at $1500 \AA$ in the rest frame to get an independent estimate of the SFR (uncorrected for extinction). Using the relation in Madau et al. (1998)

$\operatorname{SFR}\left(M_{\odot} \mathrm{yr}^{-1}\right)=1.3 \times 10^{-28} L_{v} \quad\left[\mathrm{erg} \mathrm{s}^{-1} \mathrm{~Hz}^{-1}\right]$

we find SFR $=4.3 \pm 2.0$ and $7.4 \pm 3.0 M_{\odot} \mathrm{yr}^{-1}$ not corrected for internal extinction for the two hosts, respectively. In principle, 
Table 4. SFRs calculated from ground based GRB host observations. Column 2 lists the spectroscopic redshifts and Col. 3 the corresponding luminosity distances. Columns 4 and 5 list the inferred UV flux and SFRs respectively, and Col. 6 the SFRs corrected for the internal extinction from Table 2. The intrinsic scatter of $30 \%$ for the UV to SFR calibration has not been included in the reported errors. The last two columns list the SFRs derived from spectroscopic measurements of the [O II] lines and sub-mm/radio observations taken from following references: (1) Bloom et al. (2001), (2) Bloom et al. (1998), (3) Djorgovski et al. (1998), (4) Hjorth et al. (2000a,b), (5) Castro-Tirado et al. (2001), (6) Piro et al. (2002), (7) Bloom et al. (2003), (8) Berger et al. (2003), (9) Vreeswijk et al. (2001). Note that these SFRs are sometimes derived using a different cosmology than adopted in this paper which will cause a small change in the derived SFR.

\begin{tabular}{cccccclc}
\hline \hline Host & $z$ & $\begin{array}{c}d_{L} \\
(\mathrm{~cm})\end{array}$ & $\begin{array}{c}\text { UV flux } \\
(\mu \mathrm{Jy})\end{array}$ & $\begin{array}{c}\text { SFR } \\
\left(M_{\odot} \mathrm{yr}^{-1}\right)\end{array}$ & $\begin{array}{c}\text { Unextincted SFR } \\
\left(M_{\odot} \mathrm{yr}^{-1}\right)\end{array}$ & $\begin{array}{l}\text { [O II] Based SFR (Refs. }) \\
\left(M_{\odot} \mathrm{yr}^{-1}\right)\end{array}$ & $\begin{array}{l}\text { Radio/sub-mm SFR } \\
\left(M_{\odot} \mathrm{yr}^{-1}\right)\end{array}$ \\
\hline GRB 970228 & 0.695 & $1.40 \times 10^{28}$ & $0.34 \pm 0.16$ & $0.70 \pm 0.32$ & $0.78 \pm 0.36$ & $0.76(1)$ & $<335(8)$ \\
GRB 970508 & 0.835 & $1.76 \times 10^{28}$ & $0.28 \pm 0.15$ & $0.83 \pm 0.45$ & $1.10 \pm 0.60$ & $1.4(2)$ & $<380(8)$ \\
GRB 980703 & 0.966 & $2.10 \times 10^{28}$ & $3.20 \pm 0.08$ & $12.7 \pm 0.32$ & $23.8 \pm 0.60$ & $20(3)$ & $180 \pm 25(8)$ \\
GRB 990123 & 1.600 & $3.93 \times 10^{28}$ & $0.55 \pm 0.16$ & $5.71 \pm 1.69$ & $28.0 \pm 8.29$ & & $<100(9)$ \\
GRB 990712 & 0.433 & $7.95 \times 10^{27}$ & $1.65 \pm 0.04$ & $1.28 \pm 0.03$ & $1.64 \pm 0.04$ & $2.12 \pm 0.60(4)$ & $70 \pm 30(8)$ \\
GRB 991208 & 0.706 & $1.43 \times 10^{28}$ & $0.40 \pm 0.13$ & $0.83 \pm 0.28$ & $1.54 \pm 0.52$ & $4.8 \pm 0.2(5)$ & $90 \pm 45(8)$ \\
GRB 000210 & 0.846 & $1.77 \times 10^{28}$ & $0.79 \pm 0.07$ & $2.37 \pm 0.20$ & $2.62 \pm 0.28$ & $3(6)$ & $330 \pm 75(8)$ \\
GRB 000418 & 1.118 & $2.52 \times 10^{28}$ & $1.33 \pm 0.04$ & $7.02 \pm 0.22$ & $8.85 \pm 0.22$ & $55(7)$ & $820 \pm 340(8)$ \\
GRB 000926 & 2.037 & $5.28 \times 10^{28}$ & $0.50 \pm 0.26$ & $8.09 \pm 4.16$ & $8.37 \pm 4.30$ & & \\
GRB 010921 & 0.451 & $8.32 \times 10^{27}$ & $2.15 \pm 0.08$ & $1.81 \pm 0.07$ & $5.67 \pm 0.22$ & & \\
\hline
\end{tabular}

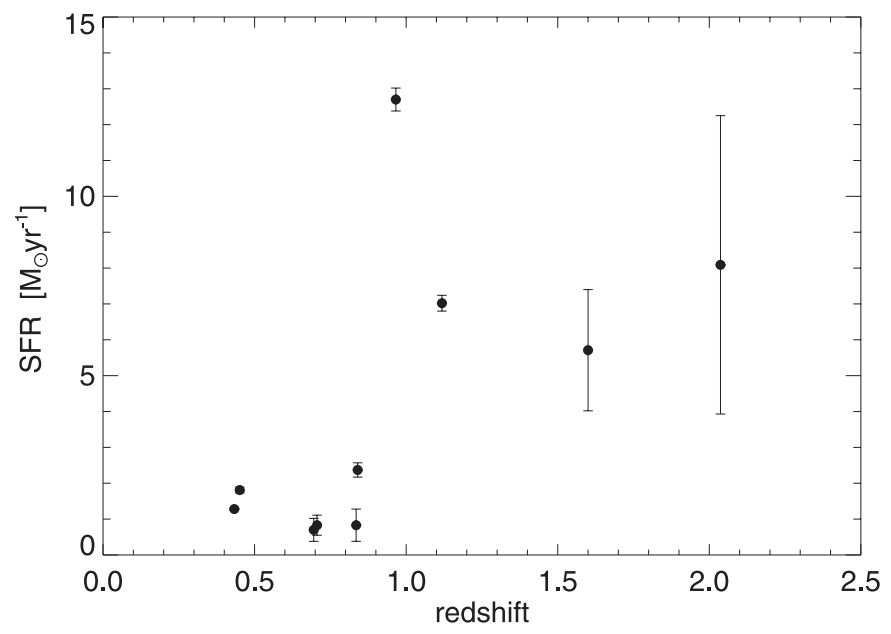

Fig. 2. Star-formation rates of the $10 \mathrm{GRB}$ hosts as a function of redshifts. The SFRs have not been corrected for the effect of host galaxy extinction. A trend of larger SFRs for the hosts at larger redshifts is very likely a selection effect.

the SFR derived from the continuum at smaller wavelengths could be used to constrain the intrinsic extinction, but in practice this is difficult given the photometric uncertainties. Indeed, one measurement appears to give a smaller SFR based on the $1500 \AA$ calibration compared to the $2800 \AA$ calibration, while the other is slightly larger. However, in both cases the SFRs are consistent within $1 \sigma$ errors.

\subsection{Specific SFRs}

Even though the SFRs appear small, they show variations of more than a factor of 10 between the individual hosts. A more informative measure of the star-formation activity in the galaxies may be the SFR per unit luminosity. The rest-frame $B$ band luminosities are calculated from the best fit (rest frame) spectra by convolving with the $B$ band filter transmission. This way, all information from the broad band observations is used, and no $\mathrm{K}$-correction is involved. Using the cosmological model we can estimate the absolute $B$ band magnitude and the corresponding luminosity of the galaxy, $L$, given in Table 5 .

It is evident that some of the hosts are less luminous than an $M^{*}$ galaxy, i.e., the magnitude of a galaxy at the break in the Schechter luminosity function (Schechter 1976). A similar conclusion based on colours of a sample of GRB hosts was reached by Le Floc'h et al. (2003). We adopt $M^{*}=-21$ which is typically inferred for field galaxies. At higher redshifts this magnitude is reported to vary for blue galaxies (Lilly et al. 1995), but since all galaxies are treated identically here the actual value of $M^{*}$ will just introduce a systematic change of the specific SFRs.

The SFRs from Table 4 were divided by the quantity $L / L^{*}$ in order to calculate specific SFRs presented in Table 5. The specific SFRs (not corrected for extinction) vary by a factor of $\sim 2$, as shown in the upper panel of Fig. 3. Choosing a different magnitude for an $M^{*}$ galaxy does not change this result. The distribution of specific SFRs has a mean of $9.7 M_{\odot} \mathrm{yr}^{-1}\left(L / L^{*}\right)^{-1}$ and a standard deviation of 2.1. Since the SFR is a measure of the flux in the UV rest frame, and the luminosity is a measure of the rest frame flux at $\sim 4400 \AA$, the specific SFR is simply characterizing the slope of the spectrum for each host.

\section{Comparison with field galaxies}

Le Floc'h et al. (2003) found that GRB hosts are rather blue compared to other high redshift galaxies, which could be interpreted as GRB hosts having higher SFRs, or being less dusty than the average galaxy. We will now investigate whether the GRB host SFRs are different from another sample of high redshift galaxies. The SFRs for the field galaxies should be derived in the same manner as for the GRB hosts. We therefore need a large sample of high redshift galaxies selected from optical methods and for which estimates of the redshifts exist. 
Table 5. Absolute magnitudes, luminosities relative to an $L^{*}$ galaxy, and specific SFRs of the GRB hosts. The specific SFRs are not corrected for internal extinction.

\begin{tabular}{lllll}
\hline \hline Host & $z$ & $M_{B}$ & $L / L^{*}$ & $\begin{array}{l}\text { Specific SFR } \\
\left(M_{\odot} \mathrm{yr}^{-1}\left(L / L^{*}\right)^{-1}\right)\end{array}$ \\
\hline GRB 970228 & 0.695 & -18.08 & 0.07 & $10.3 \pm 4.71$ \\
GRB 970508 & 0.835 & -18.14 & 0.07 & $11.6 \pm 6.27$ \\
GRB 980703 & 0.966 & -21.39 & 1.43 & $8.87 \pm 0.22$ \\
GRB 990123 & 1.600 & -21.05 & 1.05 & $5.45 \pm 1.61$ \\
GRB 990712 & 0.433 & -18.56 & 0.11 & $12.1 \pm 0.28$ \\
GRB 991208 & 0.706 & -18.48 & 0.10 & $8.45 \pm 2.85$ \\
GRB 000210 & 0.846 & -19.36 & 0.22 & $10.7 \pm 0.91$ \\
GRB 000418 & 1.118 & -20.41 & 0.58 & $12.1 \pm 0.38$ \\
GRB 000926 & 2.037 & -20.82 & 0.85 & $9.55 \pm 4.91$ \\
GRB 010921 & 0.451 & -19.42 & 0.23 & $7.83 \pm 0.35$ \\
\hline
\end{tabular}

\subsection{Comparison sample}

Magnitudes in the $U B V I J H K$ bands and photometric redshifts of 1067 galaxies in the Hubble Deep Field North (HDFN) have been estimated in Fernández-Soto et al. (1999) ${ }^{3}$. Data in the $U B V I$ bands were obtained with the WFPC2 using the broad band filters $F 300 W, F 450 W, F 606 W$, and $F 814 W$, respectively. The $J H K$ data were from ground based photometry obtained with the $4 \mathrm{~m}$ telescope at the Kitt Peak Observatory. Photometric redshifts are uncertain within $\Delta z=0.1$ for the brightest galaxies with $I<25$ (Bolzonella et al. 2000), estimated by comparing the photometric redshifts to the spectroscopic redshifts of more than 100 galaxies in the catalog. Since the galaxies have been observed in 7 bands and have smaller photometric errors the photometric redshift accuracy is better than for the GRB host sample. Additionally, a catalog containing 1611 galaxies, with optical data from WFPC2 and near-IR data from the VLT/ISAAC of the HDF South (HDFS) was included (Vanzella et al. 2001). Compared to the GRB hosts these galaxies, which we will collectively refer to as HDF galaxies, have a wider span in magnitudes and redshifts.

The flux densities in the various filters given in the catalogs were converted into $A B$ magnitudes which were used as input for HyperZ. Conversion factors were calculated using information of the throughputs of the WFPC2 filters for the optical data. Similarly, for the near-IR data the throughputs of the Kitt Peak and VLT/ISAAC filters were used, respectively.

Photometric redshifts, best fitting templates, extinctions, and absolute $B$ band magnitudes were estimated for all galaxies using the same cosmology as for the GRB hosts.

In the redshift range corresponding to the redshift distribution of the GRB hosts analysed here, $0.4<z<2.1$, the number of galaxies that have photometric redshifts differing by more than 0.2 from the spectroscopic measure is $10 \%$. This number represents a measure of the overall accuracy of the photometric redshift estimations.

The SFRs of the 1067+1611 HDF galaxies were calculated in the same way as described above for the GRB hosts. In total 1140 galaxies were categorized as starburst galaxies, and for

\footnotetext{
${ }^{3}$ http://bat.phys. unsw.edu.au/ fsoto/hdfcat.html
}
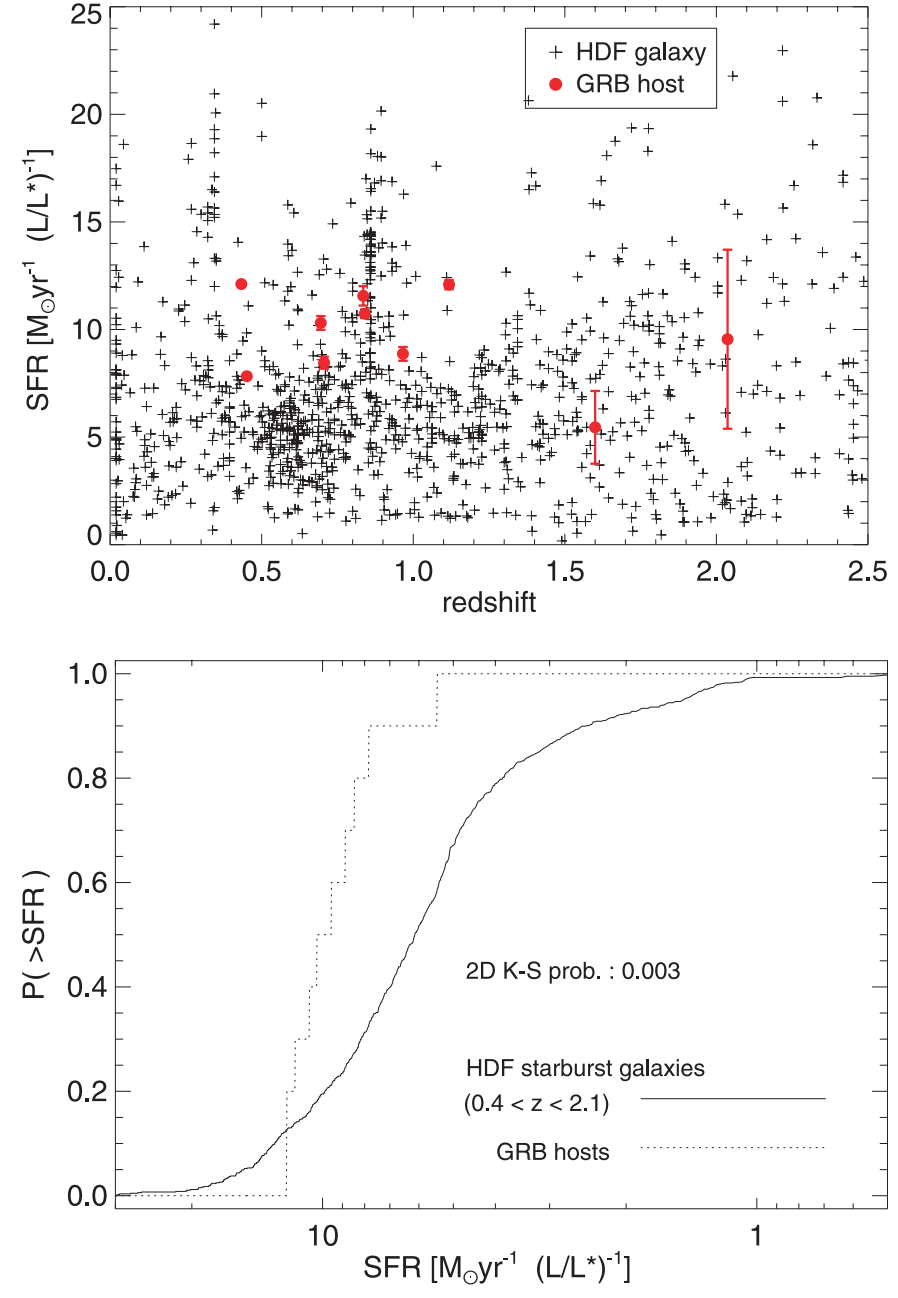

Fig. 3. Upper panel: specific SFRs for 1140 starburst galaxies in the HDF North and South. The galaxies have photometric redshifts estimated by HyperZ and are all classified as starburst galaxies. The SFRs of the 10 GRB hosts are shown as large circles. None of the SFRs are corrected for intrinsic extinction. Lower panel: cumulative distribution of the specific SFR for 851 starburst galaxies having $0.4<z<2.1$ (solid curve) and for 10 GRB hosts galaxies in the same redshift range (dotted curve). $67 \%$ of the HDF galaxies classified as starburst galaxies (573 among 851) have specific SFRs of more than $5 M_{\odot} \mathrm{yr}^{-1}\left(L / L^{*}\right)^{-1}$, which is the smallest specific SFR of all the GRB hosts.

those the specific SFRs (in $\left.M_{\odot} \mathrm{yr}^{-1}\left(L / L^{*}\right)^{-1}\right)$ were determined. As above, we assumed $M^{*}=-21$ and did not correct for the effect of extinction. The results are presented in Fig. 3. The top panel shows the specific SFRs of 1140 HDF starburst galaxies as a function of their estimated photometric redshifts. The specific SFRs (not corrected for extinction) for the GRB hosts are shown as large circles. Error bars are not included, but for each point the error is $\sim 30 \%$, due to the intrinsic scatter of the SFR estimator. All specific SFRs of the HDF galaxies are in the range $0-20 M_{\odot} \mathrm{yr}^{-1}\left(L / L^{*}\right)^{-1}$. However relatively few galaxies $(20 \%)$ have specific SFRs exceeding $10 M_{\odot} \mathrm{yr}^{-1}\left(L / L^{*}\right)^{-1}$, whereas this is the case for $50 \%$ of the GRB hosts.

To perform a direct comparison with the sample of GRB hosts, 851 HDF starburst galaxies with photometric redshifts in the range $0.4<z<2.1$ were selected. The lower 
panel in Fig. 3 shows the cumulative distribution of the specific SFRs of these galaxies. On average the specific SFRs for the GRB hosts are larger than for the field galaxies. Out of the $851 \mathrm{HDF}$ galaxies 573 galaxies have specific SFRs above $5 M_{\odot} \mathrm{yr}^{-1}\left(L / L^{*}\right)^{-1}$, which is the lower range of the SFRs of the GRB hosts. Taken at face value, this implies that the population of all GRB hosts lie among the upper $67 \%$ of starburst galaxies, implying that GRB hosts have specific SFRs which are larger than for ordinary field starburst galaxies at similar redshifts. Another explanation could be that the GRB hosts have less extinction. We shall return to a discussion of this effect in Sects. 6.4 and 6.5.

We performed a statistical test in order to determine whether the distribution of specific SFRs vs. redshifts is different for the two samples. We applied a two-dimensional, twosample Kolmogorov-Smirnov test (K-S test) (Peacock 1983) which uses two 2-dimensional samples and checks the probability that one sample has the same parent distribution as the other. We used the implementation of the test described in Fasano \& Franceschini (1987), which uses significantly fewer computations. According to Peacock (1983) the test is valid when both the sample sizes are greater than or equal to 10 . Applications of the tests, described in Fasano \& Franceschini (1987) and Peacock (1983), have shown that there is no difference for uncorrelated distributions, within statistical uncertainties, between the two tests. When the probability is $>0.2$ the value of the probability is not accurate, but the hypothesis that the two distributions are not significantly different is correct (Press et al. 1992), and the derived probabilities can be considered as lower limits.

Our qualitative finding that the distributions of specific SFRs vs. redshifts are different for the GRB hosts and HDF starburst galaxies is supported by the $2 \mathrm{D} \mathrm{K}-\mathrm{S}$ test, which gives a probability of 0.003 that the parent population is the same for the two samples. Hereafter, when a two dimensional test is performed, the first dimension corresponds to the redshift and the second to the SFRs as in the upper panel in Fig. 3. Thus, we calculate $2 \mathrm{D}$ probabilities for the distributions while showing the corresponding 1D cumulative distribution, e.g. as in the lower panel in Fig. 3.

\subsection{Population ages}

In Sect. 4 we found that GRB hosts are young starburst galaxies. We therefore investigated whether they are younger on average than field starburst galaxies. For comparison we used the ages for the 851 HDF starburst galaxies. In Fig. 4 the distribution of ages of all HDF galaxies, the GRB hosts and the HDF starburst galaxies are shown by the solid, dashed, and dotted lines, respectively.

In addition to the two-dimensional, two sample test, we also use a one-dimensional, two sample $\mathrm{K}-\mathrm{S}$ test. For the 1D two sample test, the probabilities are reliable for sample sizes $N=N_{1} N_{2} /\left(N_{1}+N_{2}\right)>4$, where $N_{1}$ and $N_{2}$ are the number of objects in sample 1 and 2 , respectively. This criterion is always satisfied for the tests performed in this paper.

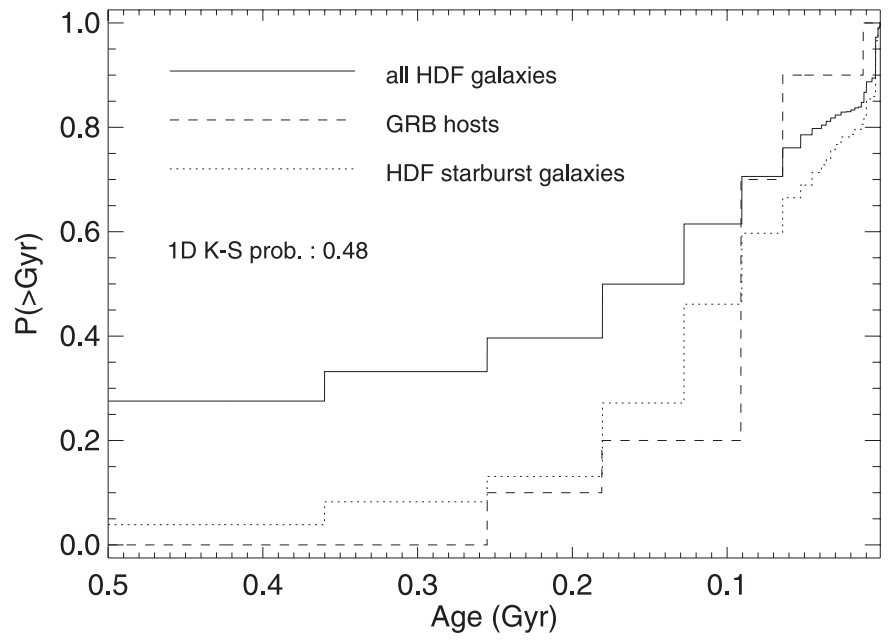

Fig. 4. Age distribution of HDF galaxies and GRB host. The solid line shows the ages for all types of HDF galaxies in the redshift range $0.4<z<2.1$, while the dotted line shows the distribution of starburst galaxies only. The step-like appearance is due to the grid of ages of the available templates. A $1 \mathrm{D} \mathrm{K}-\mathrm{S}$ test give a probability of $48 \%$ that the GRB host and HDF starburst galaxy distributions are the same.

Additionally, the probabilities are reliable in contrast with the $2 \mathrm{D}$ test.

Using a one-dimensional $\mathrm{K}-\mathrm{S}$ test on the distribution of the 851 starburst galaxies ages, we find a probability of 0.48 that the two distributions are the same, whereas a $2 \mathrm{D} \mathrm{K}-\mathrm{S}$ test gives a probability of 0.18 that the distributions of age vs. redshifts are similar for the two populations. Therefore, we have no clear indication that GRB hosts are on average younger than field starburst galaxies. We furthermore checked whether the redshift distribution of the GRB hosts and the HDF starburst galaxies were the same which is confirmed by a $1 \mathrm{D} \mathrm{K}-\mathrm{S}$ probability of $70 \%$.

Comparing the GRB host ages with those of all types of HDF galaxies at redshifts $0.4<z<2.1$ we find a $1 \mathrm{D} \mathrm{K}-\mathrm{S}$ probability of 0.02 that the distributions are similar. A comparison of the solid and the dashed lines in Fig. 4 shows that GRB hosts are indeed younger than a sample of all field galaxies.

\subsection{Very young galaxies}

The GRB hosts are classified by HyperZ as very young systems with ages smaller than $0.2 \mathrm{Gyr}$. We therefore also selected a comparison sample consisting of young (age $<0.2$ Gyr) HDF starburst galaxies. As expected, this changes the picture since the selected HDF galaxies now have larger average SFRs as indicated in the upper panel in Fig. 5. The GRB host SFRs now lie among the upper $83 \%$ of the HDF galaxies. Applying the $2 \mathrm{D} \mathrm{K}-\mathrm{S}$ test gives a probability of 0.01 for the same underlying distribution. If one compares the GRB hosts to HDF galaxies classified as having ages smaller than $0.1 \mathrm{Gyr}$, the two populations become rather similar as seen in the lower panel in Fig. 5. The 2D K-S test gives a probability of 0.20 for the same parent distribution. 

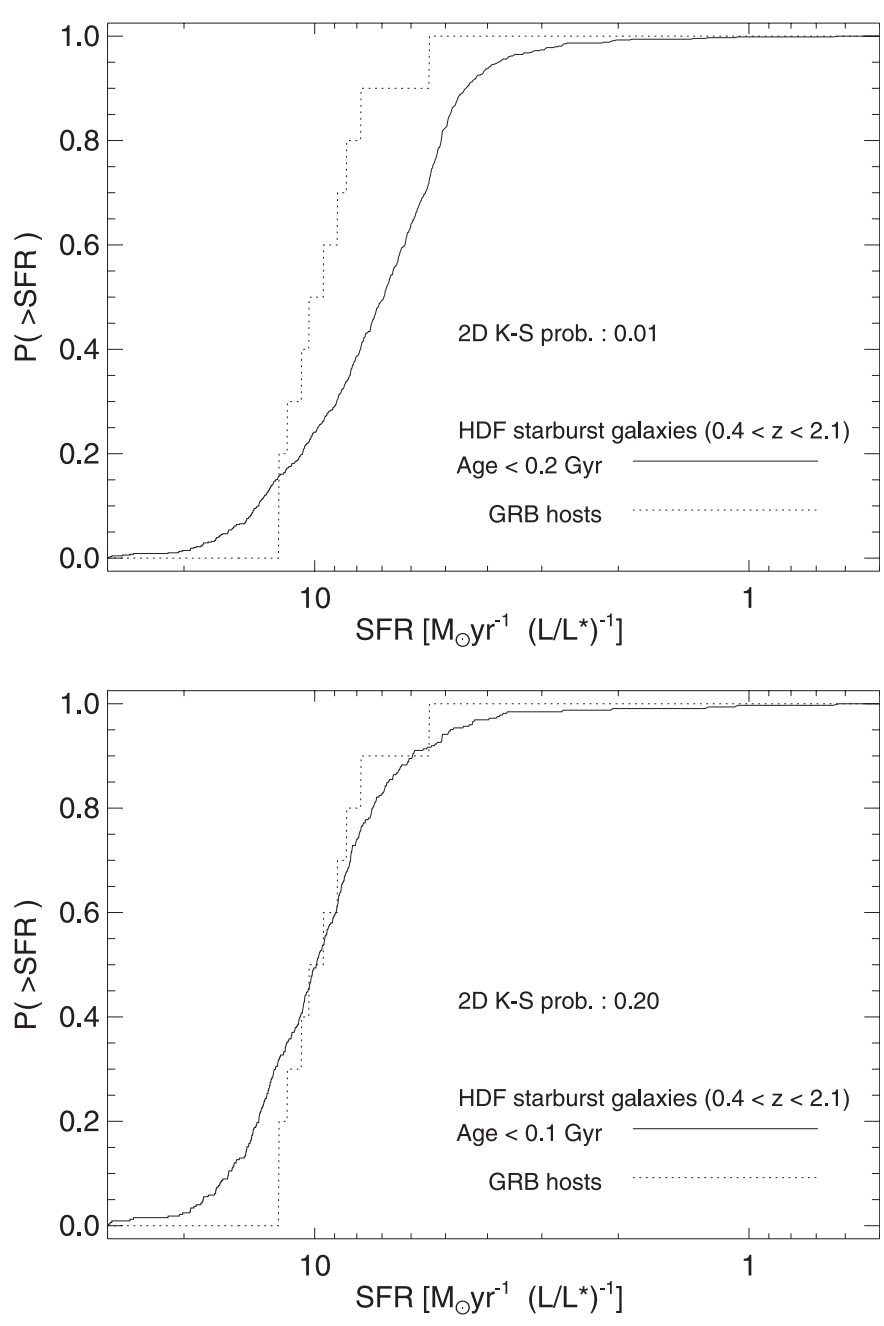

Fig. 5. These plots are similar to the one in the lower panel in Fig. 3, but here only young starburst galaxies have been included. Upper panel: the solid line represents the specific SFR distribution of $689 \mathrm{HDF}$ galaxies with ages less than $0.2 \mathrm{Gyr}$. 83\% of these HDF galaxies have specific SFRs larger than $5 M_{\odot} \mathrm{yr}^{-1}\left(L / L^{*}\right)^{-1}$. Applying the $2 \mathrm{D} \mathrm{K}-\mathrm{S}$ test gives a probability of 0.01 for the sample parent distribution. Lower panel: $327 \mathrm{HDF}$ galaxies with ages less than 0.1 Gyr for which $94 \%$ have specific SFRs larger than $5 M_{\odot} \mathrm{yr}^{-1}\left(L / L^{*}\right)^{-1}$. The two distributions are now rather similar, which is confirmed by the K-S probability of 0.20 for the same parent distribution.

These simple tests show that the specific SFRs of GRB hosts are larger on the average than a population of starburst galaxies at the same redshifts. The HDF galaxies show a wider distribution than the GRB hosts with more galaxies at the high and low end of the specific SFR distribution, as indicated by the cumulative distribution shown in the lower panel of Fig. 5.

Our finding that GRB hosts have larger specific starformation rates than field galaxies gives observational support to the selection criteria of potential GRB hosts based on numerical simulations Courty et al. (2004). Their selected GRB hosts do not have large absolute SFRs, but have high star formation efficiencies in agreement with our results.
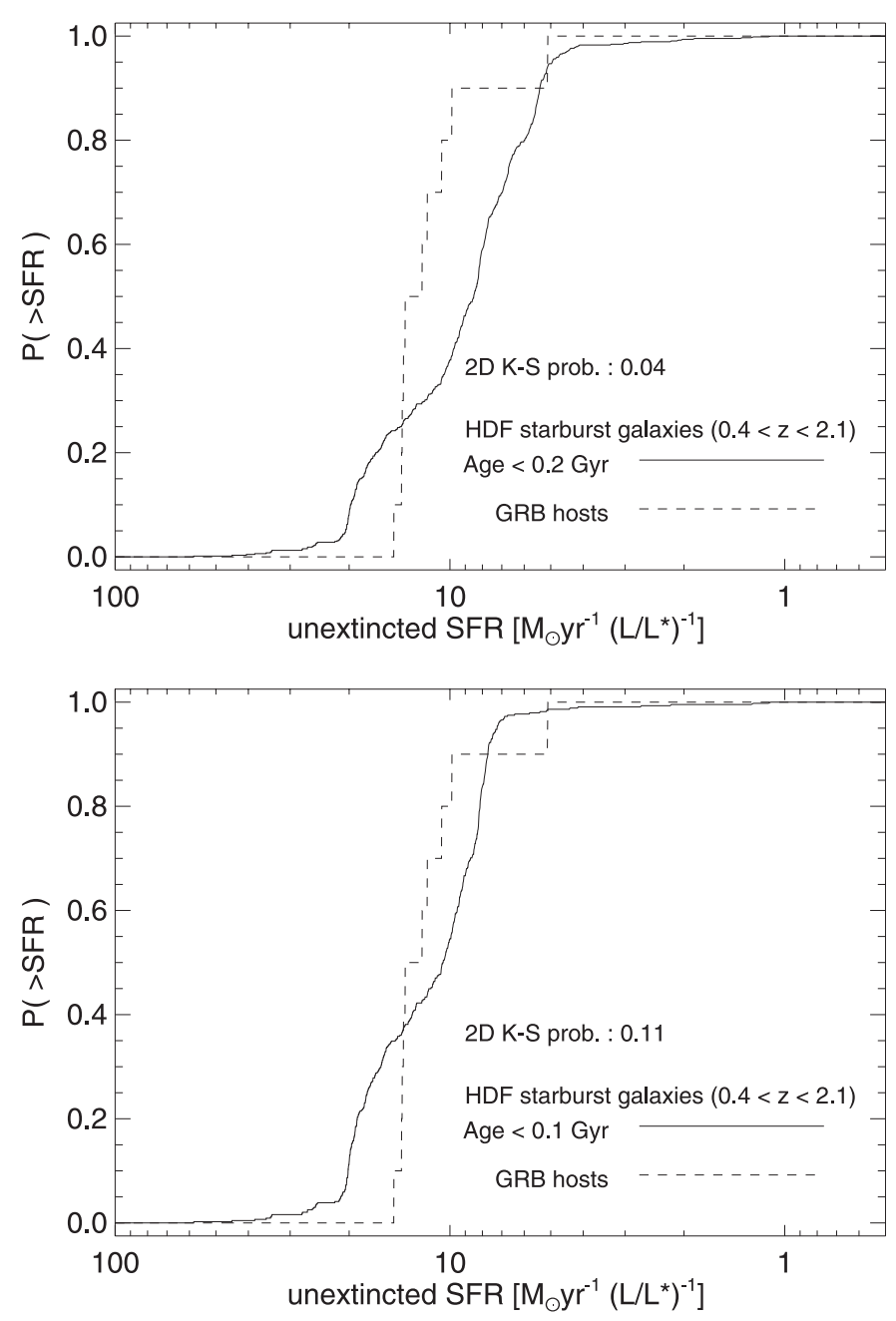

Fig. 6. Cumulative distribution of the extinction corrected specific SFRs of the HDF starburst galaxies with $0.4<z<2.1$. The upper panel consists of HDF galaxies with ages smaller than 0.2 Gyr and the lower panel of galaxies with ages smaller than 0.1 Gyr. This again shows that GRB hosts are more likely to have similar specific SFRs as very young field starburst galaxies. The one dimensional $\mathrm{K}-\mathrm{S}$ test gives the probabilities of 0.04 and 0.11 for the same distribution in the two plots, respectively.

\subsection{Comparisons with extinction corrected SFRs}

The SFRs in Table 5 were corrected for extinction and analysed in the same manner as above. Similarly, the SFRs for the HDF galaxies were corrected using the $A_{\mathrm{V}} \mathrm{s}$ estimated by HyperZ. The absolute $B$ band magnitudes of both galaxy samples were also corrected for extinction, using $R_{4400}=5.06$ for a Calzetti extinction curve $\left(A_{4400}=R_{4400} E_{B-V}\right)$. Figure 6 shows the cumulative distributions of these unextincted SFRs, where the selection criteria are the same as in Fig. 5. The upper panel shows a comparison of GRB hosts with young starburst (ages less than $0.2 \mathrm{Gyr}$ ) HDF galaxies. The $2 \mathrm{D} \mathrm{K}-\mathrm{S}$ test gives a probability of 0.04 for the same parent distribution. The lower panel shows the younger (age $<0.1 \mathrm{Gyr}$ ) HDF galaxies, and the $2 \mathrm{D} \mathrm{K}-\mathrm{S}$ test gives a probability of 0.11 for the same parent distribution. 


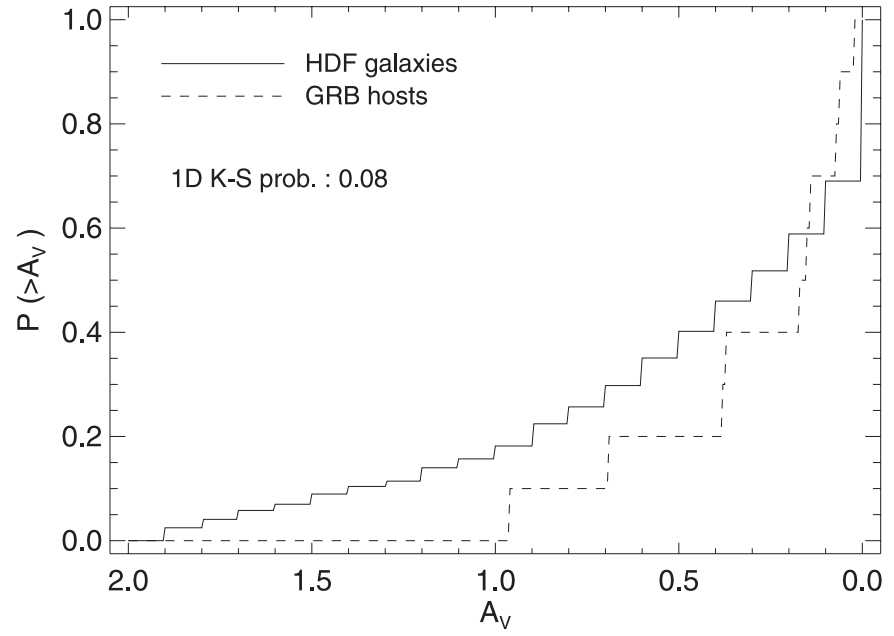

Fig. 7. Cumulative distribution of the extinction values found for the GRB hosts (dotted line) and young $(<0.1 \mathrm{Gyr})$ HDF field galaxies (solid line). In both samples a low extinction $A_{\mathrm{V}}<2$ is preferred by the best fits of the SEDs. The two distributions appear different from each other, where the GRB hosts extinctions are on average smaller than for field galaxies, but performing $1 \mathrm{D} \mathrm{K}-\mathrm{S}$ tests on the distributions gives inconclusive probabilities.

All the calculated probabilities are given in Table 6, using either Miller \& Scalo or Salpeter based templates. Generally, the differences between the two are small. We can therefore conclude that GRB hosts are not drawn at random from the average field starburst galaxy population and the GRB hosts are most likely similar to HDF starburst galaxies with very young $(t<0.1 \mathrm{Gyr})$ stellar populations. This conclusion is independent on the assumed IMF and extinction correction.

\subsection{Intrinsic extinction}

The probabilities for the extinction corrected SFR distributions are generally smaller than for the same uncorrected SFR distributions which could indicate that the extinctions found for the GRB hosts are different from those of young field galaxies. In Fig. 7 we show the cumulative distributions of the extinction values found for the two samples. Extinction values for the field galaxies are from the young (age $<0.1 \mathrm{Gyr}$ ) HDF galaxies. It seems that the distributions are different in the sense that GRB hosts have on average smaller extinctions compared to the young field galaxies. However, performing one-dimensional $\mathrm{K}-\mathrm{S}$ tests on the distributions of extinction values yields probabilities of 0.29 and 0.08 , for the same distribution, in the case of the extinctions derived from a Miller \& Scalo IMF and a Salpeter IMF, respectively. Therefore, the small GRB host sample does not allow us to determine whether or not GRB hosts have smaller intrinsic extinctions than young field starburst galaxies. Likewise, comparing GRB host extinctions with those derived for all types of field galaxies does not allow us to determine whether they have different extinction distributions.

\subsection{Additional tests}

As mentioned in Sect. 4 the SED fit of the host of GRB 000418 could indicate a somewhat larger extinction $\left(A_{\mathrm{V}}=1.4\right)$. We therefore investigated the impact of changing this particular extinction value on the derived probabilities for the extinction corrected specific SFRs. For a Salpeter IMF, the derived 2D K-S probabilities for the same parent distribution are 0.03 and 0.04 for 0.2 Gyr and 0.1 Gyr old HDF starburst, respectively, while Table 6 gives 0.004 and 0.04 . We therefore conclude that the uncertainty in the GRB 000418 host extinction has little impact on the results.

We also investigated the possibility that other galaxy types might have similar SFRs as the GRB hosts. Selecting young HDF galaxies classified as other spectral types than starbursts, i.e. irregular galaxies or spirals with $t<0.1 \mathrm{Gyr}$, gives a 2D K-S probability of 0.002 for the same parent distribution. We note that for the theoretical templates the differences between the templates at such young ages are small. However, comparing the specific extinction corrected SFRs with those of ellipticals, without any age constraint, the probability is also small $\left(2 \times 10^{-5}\right)$ which is not surprising as old elliptical galaxies do not have any star formation.

Because of possible differences between bright and faint HDF galaxies, an additional brightness requirement on the HDF galaxies was therefore imposed. We selected HDF galaxies with absolute $B$ band magnitudes as bright or brighter than GRB hosts $\left(M_{B}<-18.08\right)$ and the resulting $\mathrm{K}-\mathrm{S}$ probabilities for the various tests are listed in Cols. 4 and 5 in Table 6. The probabilities now appear to be much smaller than without the brightness selection (apart from the M\&S IMF based extinction corrected tests). This is not due to the smaller number of comparison galaxies, but arises because the distribution of specific SFRs for the GRB hosts is narrower than for the HDF galaxies as also seen in Figs. 5 and 6. However, we consider that an increase in the GRB host sample is necessary before this difference in the distributions can be explained.

\section{Discussion and conclusion}

We have constructed a flux-limited sample $(R<25.3)$ of all GRB host galaxies with known redshifts. The sample consists of 10 galaxies with broad-band magnitudes in more than 5 filters obtained from the literature. The GRBs which occurred in these galaxies comprise a collection of a dark burst (GRB 000210), a dim one (GRB 000418), a bright one (GRB 990712), and a very bright one (GRB 990123).

Comparing the SEDs of the GRB hosts with template spectra we find that they are young starburst galaxies with moderate to low extinctions $\left(A_{\mathrm{V}}<1\right)$. Photometric redshifts are found to be accurate, with a standard deviation of \pm 0.21 from the spectroscopic ones. Accurate photometric redshifts are obtained provided there is sufficient optical-IR coverage and the magnitudes are accurate to the 10-20\% level. Through the analysis of the SEDs of GRB hosts we have found that it is important to include near-IR magnitudes when estimating the extinction, since the effect of extinction is largest in the UV region, 
Table 6. 2-dimensional K-S probabilities for the GRB hosts having the same parent distribution of specific SFRs vs. redshifts as the starburst HDF galaxies at similar redshifts $(0.4<z<2.1)$ with the ages listed in Col. 1 . In brackets are given the number of galaxies which are used for comparison. Columns 2 and 3 impose no selection criteria on the brightness of the comparison galaxies, while Cols. 4 and 5 include an additional criterion, that the HDF galaxies be as bright or brighter than absolute $B$ band magnitude of the GRB hosts.

\begin{tabular}{lll|ll}
\hline \hline Age (Gyr) & M\&S IMF & Salpeter IMF & M\&S IMF & Salpeter IMF \\
\hline All & $0.003(851)$ & $0.006(823)$ & $0.0001(111)$ & $0.0005(128)$ \\
$<0.2$ & $0.01(689)$ & $0.02(692)$ & $0.0008(85)$ & $0.007(80)$ \\
$<0.1$ & $0.20(464)$ & $0.24(444)$ & $0.003(46)$ & $0.002(42)$ \\
\hline Extinction corrected & & & \\
\hline All & $0.006(783)$ & $0.001(794)$ & $0.05(164)$ & $0.001(218)$ \\
$<0.2$ & $0.04(642)$ & $0.004(676)$ & $0.06(131)$ & $0.006(164)$ \\
$<0.1$ & $0.11(439)$ & $0.04(440)$ & $0.008(84)$ & $0.004(95)$ \\
\hline
\end{tabular}

and a better sampling of the broad band SEDs of the hosts gives a more secure estimate of the extinction.

By comparing extinctions derived from the SEDs with those of galaxies in the HDF North and South, we found that the intrinsic extinctions of the GRB hosts are small and not significantly different on average from those of either young field starburst galaxies or field galaxies in general.

The coincidence between small values of $A_{\mathrm{V}}$ from the host SED and that of the afterglow suggests that we mostly see effects of the global extinction in the afterglow light curves. Moreover, small extinction values do not exclude the possibility that the GRBs themselves are located in more dusty and higher density environments, such as embedded in molecular clouds which has been suggested through analyses of X-ray afterglows. This was discussed for the case of the GRB 000210 host galaxy, where a large $N_{\mathrm{H}}$ was inferred (Piro et al. 2002), while the galaxy itself shows a small global extinction (see Table 2; and Gorosabel et al. 2003a). However, if the regions of star formation where GRBs occur are small and not numerous, this will not have a large effect on the overall SEDs of the hosts.

For all host galaxies the inferred ages are less than $0.2 \mathrm{Gyr}$ while most galaxies have even younger populations, $t<$ 0.1 Gyr. A comparison of GRB host ages with those of HDF galaxies showed that GRB hosts are not significantly younger than starburst field galaxies at similar redshifts, but are clearly younger than a sample of all types of field galaxies.

A good sampling of the redshifted Balmer jump/4000 $\AA$ break gives a well determined age for the dominant population of stars in the galaxy. With multiband photometry this jump is sampled well for all galaxies in the redshift range involved in this study which indicates that the ages of the dominant population of stars are well constrained. Through fits to templates of various metallicities we find that different template metallicities give consistent estimates for the derived best fit ages. Specifically, for 2 hosts the ages varied by a factor of $\lesssim 2$, while for the 8 remaining hosts, the ages were consistent.

The SFRs of the hosts were calculated from their rest frame $2800 \AA$ flux and was found to vary by more than an order of magnitude from host to host. Specific SFRs, obtained by normalising the SFRs with respect to the luminosities of the hosts are more clustered around the mean value ranging from 5 to
$12 M_{\odot} \mathrm{yr}^{-1}\left(L / L^{*}\right)^{-1}$ independently of the redshift. Comparing these with specific SFRs of high redshift galaxies in the HDF we found that the specific SFRs for GRB hosts lie among the upper $66 \%$ of the specific SFRs for the field galaxies in the same redshift range $(0.4<z<2.1)$. We performed several two-dimensional $\mathrm{K}-\mathrm{S}$ tests to quantify the comparisons of GRB hosts and subsets of the HDF field galaxy sample. We found that GRB hosts most likely have specific SFRs similar to very young field galaxies with ages less than 0.1 Gyr. Taking extinction effects into account does not change this result. The inferred young ages of the dominant stellar populations of the GRB hosts are in agreement with the idea that GRBs are associated with core collapse SNe (Woosley 1993; Galama et al. 1998; Hjorth et al. 2003b).

We have found that GRB hosts are not younger than field starburst galaxies but have similar specific SFRs as the youngest starburst galaxies showing that GRB hosts belong to a group of very young, actively star forming galaxies.

The ages are inferred from the size of the Balmer jump/4000 $\AA$ break, but also from the slope of the spectrum, while the specific SFRs measure the flux ratio between $4400 \AA$ and $2800 \AA$ in the rest frame. All SED fits were done with templates of solar metallicity, which is likely a simplification.

Some GRB hosts are found to be Lyman $\alpha$ emitters indicating that these galaxies contain only little dust or have low metallicities (Fynbo et al. 2003). Low dust content and low metallicity of the environment is also indicated by spectroscopic observations of the optical afterglow of GRB 020124 (Hjorth et al. 2003a). Low internal metallicity for GRB hosts would imply that we observe bluer colours relative to the HDF galaxies which give rise to larger specific SFRs and furthermore, the SED fits would result in a younger age assuming solar metallicity. The conclusions that GRB hosts have similar ages as field starburst galaxy and yet appear to have larger specific SFRs may therefore be consistent.

A larger sample of GRB hosts can be constructed by obtaining multiband observations of hosts of bursts which have occurred within the past two years. This can be used to analyse SEDs and infer SFRs from individual galaxies along the lines presented in this paper. Moreover, with future space based missions, such as Swift, a sample of uniformly selected GRBs with sufficiently brights host galaxies is within reach. 
This would allow a detailed quantitative comparison with the properties of specific subsamples of optically selected field galaxies at high redshift.

Acknowledgements. L. Christensen acknowledges support by the German Verbundforschung associated with the ULTROS project, grant No. 05AE2BAA/4. We are grateful to Johan Fynbo for providing a preliminary GRB 000926 near-infrared host magnitude prior to publication. The authors acknowledge benefits from collaboration with the EU FP5 Research Training Network "Gamma Ray Bursts: An Enigma and a Tool". This work was supported by the Danish Natural Science Research Council (SNF). We finally thank the anonymous referee for comments and suggestions which helped to improve the paper.

\section{References}

Andersen, M. I., Hjorth, J., Pedersen, H., et al. 2000, A\&A, 364, L54 Berger, E., Kulkarni, S. R., \& Frail, D. A. 2001, ApJ, 560, 652

Berger, E., Kulkarni, S. R., Bloom, J. S., et al. 2002, ApJ, 581, 981

Berger, E., Cowie, L. L., Kulkarni, S. R., et al. 2003, ApJ, 588, 99

Bloom, J. S., Djorgovski, S. G., Kulkarni, S. R., \& Frail, D. A. 1998, ApJ, 507, L25

Bloom, J. S., Kulkarni, S. R., Djorgovski, S. G., et al. 1999, Nature, 401, 453

Bloom, J. S., Djorgovski, S. G., \& Kulkarni, S. R. 2001, ApJ, 554, 678

Bloom, J. S., Kulkarni, S. R., \& Djorgovski, S. G. 2002, AJ, 123, 1111

Bloom, J. S., Berger, E., Kulkarni, S. R., Djorgovski, S. G., \& Frail, D. A. 2003, AJ, 125, 999

Bolzonella, M., Miralles, J.-M., \& Pelló, R. 2000, A\&A, 363, 476

Bruzual, A. G., \& Charlot, S. 1993, ApJ, 405, 538

Bruzual, A. G., \& Charlot, S. 2003, MNRAS, 344, 1000

Calzetti, D., Armus, L., Bohlin, R. C., et al. 2000, ApJ, 533, 682

Castro, S., Galama, T. J., Harrison, F. A., et al. 2003, ApJ, 586, 128

Castro-Tirado, A. J., \& Gorosabel, J. 1999, A\&AS, 138, 449

Castro-Tirado, A. J., Zapatero-Osorio, M. R., Caon, N., et al. 1999, Science, 283, 2069

Castro-Tirado, A. J., Sokolov, V. V., Gorosabel, J., et al. 2001, A\&A, 370, 398

Chary, R., Becklin, E. E., \& Armus, L. 2002, ApJ, 566, 229

Christensen, L., Hjorth, J., Gorosabel, J., et al. 2004, A\&A, 413, 121

Courty, S., Björnsson, G., \& Gudmundsson, E. H. 2004, MNRAS, in press

de Grijs, R., Fritze-v. Alvensleben, U., Anders, P., et al. 2003, MNRAS, 342, 259

Djorgovski, S. G., Bloom, J. S., \& Kulkarni, S. R. 2003, ApJ, 591, L13

Djorgovski, S. G., Frail, D. A., Kulkarni, S. R., et al. 2001a, ApJ, 562, 654

Djorgovski, S. G., Kulkarni, S. R., Bloom, J. S., et al. 2001b, in Gamma-ray Bursts in the Afterglow Era, ed. E. Costa, F. Frontera, \& J. Hjorth (Berlin Heidelberg: Springer), 218

Djorgovski, S. G., Kulkarni, S. R., Bloom, J. S., et al. 1998, ApJ, 508, L17

Fasano, G., \& Franceschini, A. 1987, MNRAS, 225, 155

Fernández-Soto, A., Lanzetta, K. M., Chen, H., Pascarelle, S. M., \& Yahata, N. 2001, ApJS, 135, 41

Fernández-Soto, A., Lanzetta, K. M., \& Yahil, A. 1999, ApJ, 513, 34 Fitzpatrick, E. L. 1986, AJ, 92, 1068
Fruchter, A. S., Pian, E., Thorsett, S. E., et al. 1999a, ApJ, 516, 683 Fruchter, A. S., Thorsett, S. E., Metzger, M. R., et al. 1999b, ApJ, 519, L13

Fynbo, J. P. U., Jakobsson, P., Møller, P., et al. 2003, A\&A, 406, L63

Fynbo, J. U., Holland, S., Andersen, M. I., et al. 2000, ApJ, 542, L89

Galama, T. J., Vreeswijk, P. M., van Paradijs, J., et al. 1998, Nature, 395,670

Galama, T. J., Tanvir, N., Vreeswijk, P. M., et al. 2000, ApJ, 536, 185

Garnavich, P. M., Stanek, K. Z., Wyrzykowski, L., et al. 2003, ApJ, 582,924

Gorosabel, J., Christensen, L., Hjorth, J., et al. 2003a, A\&A, 400, 127 Gorosabel, J., Klose, S., Christensen, L., et al. 2003b, A\&A, 409, 123 Greiner, J., Klose, S., Salvato, M., et al. 2003, ApJ, 599, 1223

Hjorth, J., Holland, S., Courbin, F., et al. 2000a, ApJ, 539, L75

Hjorth, J., Holland, S., Courbin, F., et al. 2000b, ApJ, 534, L147

Hjorth, J., Thomsen, B., Nielsen, S. R., et al. 2002, ApJ, 576, 113

Hjorth, J., Møller, P., Gorosabel, J., et al. 2003a, ApJ, 597, 699

Hjorth, J., Sollerman, J., Møller, P., et al. 2003b, Nature, 423, 847

Holland, S., Fynbo, J. P. U., Hjorth, J., et al. 2001, A\&A, 371, 52

Holland, S., \& Hjorth, J. 1999, A\&A, 344, L67

Jakobsson, P., Hjorth, J., Fynbo, J. P. U., et al. 2003, A\&A, 408, 941

Jaunsen, A. O., Andersen, M. I., Hjorth, J., et al. 2003, A\&A, 402, 125

Kennicutt, R. C. 1998, ARA\&A, 36, 189

Kulkarni, S. R., Frail, D. A., Wieringa, M. H., et al. 1998, Nature, 395, 663

Le Floc'h, E., Duc, P.-A., Mirabel, I. F., et al. 2003, A\&A, 400, 499

Lilly, S. J., Tresse, L., Hammer, F., Crampton, D., \& Le Fevre, O. 1995, ApJ, 455, 108

Madau, P., Pozzetti, L., \& Dickinson, M. 1998, ApJ, 498, 106

Malesani, D., Tagliaferri, G., Chincarini, G., et al. 2004, ApJ, 609, L5

Miller, G. E., \& Scalo, J. M. 1979, ApJS, 41, 513

Park, H. S., Williams, G. G., Hartmann, D. H., et al. 2002, ApJ, 571, L131

Peacock, J. A. 1983, MNRAS, 202, 615

Piro, L., Frail, D. A., Gorosabel, J., et al. 2002, ApJ, 577, 680

Press, W. H., Teukolsky, S. A., Vetterling, W. T., \& Flannery, B. P. 1992, Numerical recipes in FORTRAN. The art of scientific computing (Cambridge: University Press), 2nd edition

Prevot, M. L., Lequeux, J., Prevot, L., Maurice, E., \& Rocca-Volmerange, B. 1984, A\&A, 132, 389

Price, P. A., Kulkarni, S. R., Berger, E., et al. 2002, ApJ, 571, L121

Prochaska, J. X., Bloom, J. S., Chen, H., et al. 2004, ApJ, in press

Salpeter, E. E. 1955, ApJ, 121, 161

Schechter, P. 1976, ApJ, 203, 297

Schlegel, D. J., Finkbeiner, D. P., \& Davis, M. 1998, ApJ, 500, 525

Seaton, M. J. 1979, MNRAS, 187, 73P

Sokolov, V. V., Fatkhullin, T. A., Castro-Tirado, A. J., et al. 2001, A\&A, 372, 438

Stanek, K. Z., Matheson, T., Garnavich, P. M., et al. 2003, ApJ, 591, L17

Tanvir, N. R., Barnard, V. E., Blain, A. W., et al. 2004, MNRAS, in press

Vanzella, E., Cristiani, S., Saracco, P., et al. 2001, AJ, 122, 2190

Vreeswijk, P. M., Fender, R. P., Garrett, M. A., et al. 2001, A\&A, 380, L21

Vreeswijk, P. M., Galama, T. J., Owens, A., et al. 1999, ApJ, 523, 171 Woosley, S. E. 1993, ApJ, 405, 273 